\title{
Invariance under outer inverses
}

\author{
R.E. Hartwig* P. Patrício ${ }^{\dagger}$
}

\begin{abstract}
We shall use the minus partial order combined with Pierce's decomposition to derive the class of outer inverses for idempotents, units and group invertible elements. Subsequently we show, for matrices over a field $\mathbb{F}$, that the triplet $B \hat{A} C$ is invariant under all choices of outer inverses of $A$ if and only if $B=0$ or $C=0$.
\end{abstract}

Keywords: Invariance, outer inverses, regularity, Pierce decomposition

AMS classification: 15A09, 16E50

\section{Introduction}

Let $R$ be a ring with 1 .

An element $a$ is called regular if $a a^{-} a=a$ for some inner or 1-inverse $a^{-}$. The condition for regularity is a linear condition, and the set of all inner inverses is given by

$$
\left\{a^{(1)}\right\}=a^{-}+\left(1-a^{-} a\right) R+R\left(1-a a^{-}\right) .
$$

An outer or 2-inverse $\hat{a}$ of an element $a$ is such that $\hat{a} a \hat{a}=\hat{a}$. It is a quadratic condition in $\hat{a}$. It is clear that $a \hat{a} a$ will always be regular.

A 1-2 or reflexive inverse of $a$ is denoted by $a^{+}$and satisfies

$$
a a^{+} a=a \text { and } a^{+} a a^{+}=a^{+} \text {. }
$$

\footnotetext{
*Mathematics Department, N.C.S.U., Raleigh, NC 27695-8205, U.S.A. e-mail: hartwig@unity.ncsu.edu

${ }^{\dagger}$ CMAT - Centro de Matemática and Departamento de Matemática e Aplicações, Universidade do Minho, 4710-057 Braga, Portugal. e-mail: pedro@math.uminho.pt

Partially supported by FCT- 'Fundação para a Ciência e a Tecnologia', within the project UID-MAT$00013 / 2013$.

Corresponding author.
} 
The set of all outer inverses of an element $a$ will be denoted by $T_{a}$ or $\left\{a^{(2)}\right\}$ and the set of all idempotents will be denoted by $E$. It is clear that a regular element $a$ admits $a^{-} a a^{-}$ as an outer inverse.

Given the quadratic nature of the outer inverse condition, the characterization of $T_{a}$ remains clouded in general. We shall characterize $T_{a}$ for several special types of elements, such as idempotents and units. We then use these results to nail down $T_{a}$ for group invertible elements.

We shall also use the concepts of a unit-regular element $a$, for which there is a unit $u$ in $R$ such that $a u a=a$, and (ii) that of a prime ring, for which $a R b=(0)$, forces either $a=0$ or $b=0$. It should be noted that $a$ is unit regular if and only if $a=p e q$, for some unit $p$ and $q$ and some idempotent $e$.

\section{Classes of outer inverses}

We begin by recalling [7] that for any $p, a$ and $q$, and any $\widehat{(q a p)}$

$$
p \widehat{p(q a p)} q \cdot a \cdot p \widehat{(q a p)} q=p \widehat{(q a p)} q
$$

so that it is prudent to define, for a fixed $p$ and $q$,

Definition 2.1. $S_{p, q}=\{\widehat{(q a p)} q$; any $\widehat{(q a p)}\}$

Clearly

$$
S_{p, q}=p \cdot T_{q a p} \cdot q \subseteq T_{a}=S_{1,1} .
$$

We next turn to the set of all outer inverses of 1 . It precisely equal the set of all idempotents $E$, since $x \cdot 1 \cdot x=x$ if and only if $x$ is idempotent.

Next we recall that for two units $p$ and $q$

$$
T_{p a q}=q^{-1} T_{a} p .^{-1}
$$

To characterize $T_{e}$ where $e$ is idempotent, we make use of the minus order as defined in [3] for a regular element $a$

$$
a \leq b \text { iff } a^{-} a=a^{-} b \text { and } a a^{-}=b a^{-} \text {, for some inner inverse } a^{-} .
$$

The key fact that we need is that given $e^{2}=e$ and $g$ regular such that $g \leq e$ then $g^{2}=g=g e=e g$. This also tells us that

Lemma 2.1. The following are equivalent, for $e^{2}=e$ and $g$ regular:

(i) $g \leq e$. 
(ii) $g=g e=e g=g^{2}$.

(iii) $g=e \hat{e} e$ for some outer inverse $\hat{e}$.

(iv) $g=e x e=g^{2}$ for some $x$.

We use this in

Theorem 2.1. If $e$ is idempotent then $T_{e}=v(x) g w(y)$, where $v(x)=1+(1-e) x e$, $w(y)=1+e y(1-e), g \leq e$, and $x$ and $y$ are arbitrary.

Proof. Observe that $v(x)$ and $w(y)$ are units for all $x$ and $y$ and that $e v(x)=e=w(y) e$. As such $v(x) g w(y) \cdot e \cdot v(x) g w(y)=v g e g w=v g w$. On the other hand, if $t=\hat{e}$ is any outer inverse of $e$, then we may take $x=y=t$, and select $g=e t e$. We then get

$$
\begin{aligned}
v(t) \text { etew }(t) & =[\text { ete }+(1-e) t e][1+e t(1-e)] \\
& =\text { ete }+(1-e) t e+e t(1-e)+(1-e) t(1-e) \\
& =t
\end{aligned}
$$

which is the Pierce Decomposition of $t$.

The result of Theorem 2.1 is a special case of the following [4]:

Theorem 2.2. The following are equivalent for two regular elements a and $b$ :

(i) $a \leq b$.

(ii) $a=b \hat{b} b$ for some outer inverse $\hat{b}$.

(iii) $a=b-b \hat{\hat{b}} b$ for some outer inverse $\hat{\hat{b}}$.

In which case $\hat{b} a \hat{b}=\hat{b}$ and $\hat{\hat{b}} a=0=a \hat{\hat{b}}$.

Proof. (i) implies (ii). Note that $a \leq b$ is equivalent to $a=b a^{-} a=a a^{-} b$. By taking $\hat{b}=a^{-} a a^{-}$, which is a 2-inverse of $b$, we have $b a^{-} a a^{-} b=a a^{-} b=a$.

(ii) implies (i). From $a=b \hat{b} b$ it follows $\hat{b}$ is a 1-inverse of $a$, and we may take $a^{-}=\hat{b}$. The equalities $a \hat{b}=b \hat{b}$ and $\hat{b} a=\hat{b} b$ show that $a \leq b$.

(iii) implies (ii). It is easy to show that $\hat{b}=b^{+}-b^{+} b \hat{b} b b^{+}$is indeed a 2-inverse of $b$, for any choice of a reflexive inverse $b^{+}$of $b$ and any outer inverse $\hat{\hat{b}}$. Furthermore, $b\left(b^{+}-b^{+} b \hat{\hat{b}} b b^{+}\right) b=b-b \hat{\hat{b}} b=a$.

(ii) implies (iii). Since $\hat{\hat{b}}=b^{+}-b^{+} b \hat{b} b b^{+}$is a 2-inverse of $b$, substituting in (iii) we have $b-b\left(b^{+}-b^{+} b \hat{b} b b^{+}\right) b=b-b+b \hat{b} b=a$. The remaining parts are clear. 
In general

$$
b \leq a \nRightarrow T_{b} \subseteq T_{a},
$$

as seen from the example where $b=\left[\begin{array}{ll}1 & 0 \\ 0 & 0\end{array}\right]$ and $\mathrm{a}=I_{2}$. If we pick $c=\left[\begin{array}{ll}1 & 1 \\ 1 & 1\end{array}\right]$ then $c \in T_{b}$ yet $c \notin T_{a}$.

The idempotent result of Theorem 2.1 parallels the $(2 \times 2)$ matrix case, which uses a Pierce-like decomposition. Indeed if $e=\left[\begin{array}{ll}1 & 0 \\ 0 & 0\end{array}\right]$ and $\hat{e}=\left[\begin{array}{ll}p & r \\ q & s\end{array}\right]$ then $p^{2}=p, q=x p$, and $r=p y$, so that

$$
\hat{e}=\left[\begin{array}{cc}
p & p y \\
x p & q r
\end{array}\right]=\left[\begin{array}{ll}
1 & 0 \\
x & 1
\end{array}\right]\left[\begin{array}{ll}
p & 0 \\
0 & 0
\end{array}\right]\left[\begin{array}{ll}
1 & y \\
0 & 1
\end{array}\right]=\left[\begin{array}{l}
1 \\
x
\end{array}\right] p[1, y] .
$$

In this $x$ and $y$ are arbitrary and $p$ is an arbitrary idempotent.

We have also seen the importance of outer inverses in the Brown-McCoy transformation [6].

Pierce decomposition appear naturally in the study of outer inverses.

Lemma 2.2. Given $p, q$, not necessarily idempotent,

1. $a=q a p+q a(1-p)+(1-q) a p+(1-q) a(1-p)$.

2. $(\gamma): a=q a p+(1-q) a(1-p)$ if and only if $(\alpha): q a(1-p)+(1-q) a p=0$.

3. If $q=a x, p=x a$ then $(\alpha)$ holds if and only if axa $(1-x a)+(1-a x) a x a=0$ if and only if $(\beta): 2($ axaxa - axa $)=0$. In the case $\operatorname{char}(R) \neq 2$, these are equivalent to $a(x a x-x) a=0$.

4. $(\beta) \Rightarrow(\alpha) \Rightarrow(\gamma)$.

Proof. (4). If $x=\hat{a}, q=a \hat{a}$ and $p=\hat{a} a$ then $a \hat{a} a \hat{a} a=a \hat{a} a$.

Corollary 2.1. If $a=p e q$, with $p, q$ units and e idempotent, then

$$
T_{a}=q^{-1} T_{e} p^{-1}=q^{-1}[1+(1-e) x e] e \hat{e} e[1+e y(1-e)] p^{-1},
$$

with $x, y$ arbitrary.

Corollary 2.2. If $u$ is a unit then the set of all outer inverses of $u$ is given by $T_{u}=$ $E u^{-1}=u^{-1} E$.

Proof. It is clear that $\left(e u^{-1}\right) u\left(e u^{-1}\right)=e u^{-1}$, for any $e \in E$. Conversely, if $x u x=x$, then $(x u) 1(x u)=x u$, so that $x u=e$ must be idempotent. Consequently, $x=e u^{-1}$ and the set of all outer inverses of $u$ becomes $T_{u}=E u^{-1}$. The rest follows by symmetry. 
Corollary 2.3. If $a=u e$, where $e$ is idempotent and $u$ is a unit, then $T_{a}=T_{e} u^{-1}$.

Proof. Clearly $\hat{e} u^{-1}(u e) \hat{e} u^{-1}=\hat{e} u^{-1}$. Conversely, if $x(u e) x=x$, then $(x u) e(x u)=x u$ and hence $x u \in T_{e}$. This means that $x \in T_{e} u^{-1}$.

Corollary 2.4. If a has a group inverse $a^{\#}$ then

$$
T_{a}=\left[1+\left(1-a a^{\#}\right) x a a^{\#}\right] g\left[1+a a^{\#} y\left(1-a a^{\#}\right)\right]\left(a^{\#}+1-a a^{\#}\right),
$$

where $g \leq a a^{\#}$ and $g$ is regular.

Proof. $a=\left(a+1-a a^{\#}\right)\left(a a^{\#}\right)=u e$, where $u$ is a unit and $e$ is idempotent.

Remark. Since $g=a a^{\#} g a a^{\#}$ the product simplifies considerably.

Next we give an example of a nilpotent element.

Proposition 2.1. If $N=\left[\begin{array}{ll}0 & 1 \\ 0 & 0\end{array}\right]$ then $T_{N}=\left[\begin{array}{l}x \\ 1\end{array}\right] e[1, y]$ where $e$ is idempotent and $x$ and $y$ are arbitrary.

Proof. Equating $\left[\begin{array}{ll}a & c \\ b & d\end{array}\right]\left[\begin{array}{ll}0 & 1 \\ 0 & 0\end{array}\right]\left[\begin{array}{ll}a & c \\ b & d\end{array}\right]=\left[\begin{array}{ll}a & c \\ b & d\end{array}\right]$ gives $a b=a, b^{2}=b, c=a d$ and $d=b d$, from which the result follows.

Remark. This is a special case of the full-rank-factorization result. Indeed, if $a=b c$, with $b^{-} b=1=c c^{-}$then $c^{-} e b^{-} \in T_{a}$ for all idempotents $e$. Conversely, if $x b c x=x$, then $c x b=e$ is idempotent. This may be solved to give

$$
x=c^{-} e b^{-}+\left(1-c^{-} c\right) R+R\left(1-b b^{-}\right) .
$$

But not all of these solutions are outer inverses of $a$. The solution set reduces again to a quadratic equation!

The question of containment for sets of outer inverses can be extended to unit regular elements. Inded we have the following.

Theorem 2.3. If $a$ and $b$ are unit regular elements in a prime ring, then the following are equivalent:

(i) $T_{b} \subseteq T_{a} \quad$ (ii) $b=a, \quad$ (iii) $T_{b}=T_{a}$.

Proof. Suppose $a=p e q$ and $b=r g s$, where $e$ and $g$ are idempotent and $p, q, r$ and $s$ are units. Then $T_{a}=q^{-1} T_{e} p^{-1}$ and $T_{b}=s^{-1} T_{g} r^{-1}$. Hence $T_{b} \subseteq T_{a}$ if and only if 
$\left(q s^{-1}\right) T_{g}\left(r^{-1} p\right) \subseteq T_{e}$. Because an arbitrary element in $T_{g}$ has the form $v_{g} h w_{g}$, where $h \leq g$, $v_{g}=1+(1-g) x g, w_{g}=1+g y(1-g)$, we see that

$$
T_{b} \subseteq T_{a} \text { if and only if }\left(q s^{-1}\right)\left(v_{g} h w_{g}\right)\left(r^{-1} p\right) \cdot e \cdot\left(q s^{-1}\right)\left(v_{g} h w_{g}\right)\left(r^{-1} p\right)=q s^{-1}\left(v_{g} h w_{g}\right)\left(r^{-1} p\right) .
$$

Since $q, s, v_{g}, r, p$ and $w_{g}$ are units, and $h=g h=h g$, the latter reduces to

$$
h g w_{g}\left(r^{-1} p\right) e\left(q s^{-1}\right) v_{g} g h=h
$$

whch can be simplified to

$$
h[1+y(1-g)]\left(r^{-1} a s^{-1}\right)[1+(1-g) x] h=h, \quad \text { for all } h \leq g .
$$

Since the $x$ and $y$ are arbitrary and $h$ is any element below $g$, we may select them suitably. Selecting $x=0=y$ and $h=g$, we arrive at the first necessary condition

$$
\left(h r^{-1}\right) a\left(s^{-1} h\right)=h,
$$

which gives $g\left(r^{-1} a s^{-1}\right) g=g$. Consequently $\left(r g r^{-1}\right) a\left(s^{-1} g s\right)=r g s=b$. Next we consider the inner inverse $b^{-}=s^{-1} g r^{-1}$, and obtain

$$
b b^{-}=r g r^{-1} \text { and } b^{-} b=s^{-1} g s .
$$

We thus arrive at our first necessary condition

$$
b=b b^{-} a b^{-} b .
$$

Next we set $x=0$ in (6). This gives

$$
h y(1-g) r^{-1} a s^{-1} h=0 .
$$

Since $y$ is arbitrary and $R$ is prime this ensures that

$$
(1-g) r^{-1} a s^{-1} h=0 .
$$

Left and right multiplication by $r$ and $s$ respectively gives our second necessary condition

$$
\left(1-b b^{-}\right) a b^{-} b=0 .
$$

By symmetry (i.e. setting $y=0$ ) we obtain the third condition

$$
h r^{-1} a s^{-1}(1-g) x h=0
$$

which turns into 


$$
b b^{-} a\left(1-b^{-} b\right)=0 .
$$

Substituting (7), (10), and (12) into (6) we are left with

$$
h y(1-g) r^{-1} a s^{-1}(1-g) x h=0 .
$$

Again since $R$ is prime and selecting $h \neq 0$ we obtain

$$
(1-g) r^{-1} a s^{-1}(1-g)=0 .
$$

This collapses to

$$
\left(1-b b^{-}\right) a\left(1-b^{-} b\right)=0 .
$$

Lastly, combining equations (9), (11), (13) and (16), yields

$$
a=a b^{-} b=b b^{-} a=b b^{-} a b^{-} b=b .
$$

\section{Triplet invariance over $\mathbb{F}$}

In the theory of linear models [1] the invariance of the matrix triplet $B A^{-} C$ under all choices of inner inverses $A^{-}$is essential. It is shown there that this invariance occurs if and only if the range-row-space conditions $B=X A$ and $C=A Y$, for some $X$ and $Y$, are satisfied.

In a later paper [2], it was shown that over a prime regular ring, the invariance of the triplet $B A^{+} C$ under any choice of reflexive inverse $A^{+}$is also equivalent to the range-rowspace conditions $B=X A$ and $C=A Y$.

Here we shall examine the invariance of the triplet $b \hat{a} c$ under all choices of outer inverses $\hat{a}$. In particular we shall show that $B \hat{A} C$ is invariant over a field $\mathbb{F}$, under all choices of outer inverses $\hat{A}$, if and only if either $B=0$, or $C=0$.

We now need the following definition:

Definition 3.1. Given an idempotent $e, R$ is an e-prime ring if $b g c=0, \forall g \leq e$, implies $b=0$ or $c=0$.

Note that if $f=u^{-1} e u$ and $R$ is $e$-prime then $R$ is $f$-prime. Indeed, if $b g c=0$ for all idempotents $g \leq f$ then $b u^{-1}\left(u g u^{-1}\right) u c=0$ for all idempotents $g \leq f$, ie for all $u g u^{-1} \leq e$. Since $R$ is $e$-prime then this implies $b u^{-1}=0$ or $u c=0$, that is $b=0$ or $c=0$.

Suppose $b \hat{a} c$ is invariant under $\widehat{(\cdot)}$. Since 0 is an outer inverse of $a$, this means that $b \hat{a} c=0$ for all $\hat{a}$. If we assume that $a=p e q$, for some units $p$ and $q$ and some idempotent $e$, then we arrive at

$$
b \hat{a} c=b q^{-1} v(x) g w(y) p^{-1} c=0,
$$


for all $x, y$ and $g \leq e$, where $v(x)=1+(1-g) x g, w(y)=1+g y(1-g)$.

Since $R$ is $e$-prime we may conclude that either $b q^{-1} v(x)=0$ or $w(y) p^{-1} c=0$. That is, either $b=0$ or $c=0$.

It is clear from the normal form that a square matrix over $\mathbb{F}$ can be written as $P E Q$, where $P$ and $Q$ are invertible and $E=\operatorname{diag}\left(I_{r}, 0\right)$. So let us next show that $\mathbb{F}_{n \times n}$ is an $I$-prime ring.

We begin with

Lemma 3.1. $\mathbb{F}_{n \times n}$ is I-prime. That is, if $R E S=0$ for all choices of $E^{2}=E$, either $R=0$ or $S=0$.

Proof. Setting $R=\left[\begin{array}{lll}\mathbf{r}_{1} & \cdots & \mathbf{r}_{n}\end{array}\right]$ and $S=\left[\begin{array}{c}\mathbf{s}_{1}^{T} \\ \vdots \\ \mathbf{s}_{n}^{T}\end{array}\right]$ and taking $E=E_{k k}$ we see that $\mathbf{r}_{k} \mathbf{s}_{k}^{T}=0$ for all $k=1, \ldots, n$. Since we are over a field this ensures that either $\mathbf{r}_{k}=\mathbf{0}$ or $\mathbf{s}_{k}=0$. If $R \neq 0$, we may rearrange the columns such that $R=\left[\begin{array}{lll|lll}\mathbf{r}_{1} & \cdots & \mathbf{r}_{u} & \mathbf{0} & \cdots & \mathbf{0}\end{array}\right]$ with $\mathbf{r}_{i} \neq \mathbf{0}, i=1, \ldots, u$ and $\mathbf{s}_{j}^{T}=\mathbf{0}^{T}$ for $j=1, \ldots, u$.

Next we select $E=\mathbf{e}_{1} \mathbf{e}_{1}^{T}+\mathbf{e}_{1} \mathbf{e}_{u+v}^{T}$, which yields $R E S=\mathbf{r}_{1} \mathbf{s}_{u+v}^{T}=0$ and thus forces $\mathbf{s}_{u+v}^{T}=\mathbf{0}^{T}$ for $v=1, \ldots, n-u$. Thus $S=0$. Likewise if $S \neq 0$ then $R=0$.

Theorem 3.1. BA $\hat{A}$ is 2-invariant if and only if $B=0$ or $C=0$.

Proof. Let $A=P E Q$, where $E=\operatorname{diag}\left(I_{r}, 0\right)$. Now if $G \leq E$ then $G$ has the form $G=\operatorname{diag}(H, 0)$, where $H^{2}=H$ is $r \times r$. As such

$$
B \hat{A} C=\left(B Q^{-1} V\right) G\left(W P^{-1} C\right)=R G S=R \operatorname{diag}(H, 0) S=0
$$

where $V=I+(I-E) X E, W=I+E Y(I-E)$, for all $X, Y$ and for all $r \times r$ idempotent $H$, using Theorem 2.1. Partitioning we get

$$
\left[\begin{array}{ll}
R_{1} & R_{2}
\end{array}\right]\left[\begin{array}{cc}
H & 0 \\
0 & 0
\end{array}\right]\left[\begin{array}{l}
S_{1} \\
S_{2}
\end{array}\right]=0
$$

and

$$
R_{1} H S_{1}=0
$$

where $R_{1}$ and $S_{1}^{T}$ are $n \times r$.

We next consider two arbitrary $r \times r$ submatrices $R_{I}$ and $S_{J}$ from $R_{1}$ and $S_{1}$, respectively. Then

$$
R_{I} H S_{J}=0, \forall H=H^{2} .
$$

Hence if some $S_{J} \neq 0$, then all $R_{I}$ vanish and conversely, using Lemma 3.1. In other words, either $R_{1}=0$ or $S_{1}=0$. 
If $R_{1}=0$ then $R$ is of the form $R=B Q^{-1} V=\left[\begin{array}{ll}0 & \alpha \\ 0 & \beta\end{array}\right]$.

$V$ is of the form $I+(I-E) X E=\left[\begin{array}{ll}I & 0 \\ x & I\end{array}\right]$. Since $X$ is arbitrary, then $x$ is arbitrary.

Denote $B Q^{-1}=\left[\begin{array}{ll}a & b \\ c & d\end{array}\right]$. We then have $B Q^{-1} V=\left[\begin{array}{ll}a+b x & b \\ c+d x & d\end{array}\right]=R=\left[\begin{array}{ll}0 & \alpha \\ 0 & \beta\end{array}\right]$, from which $\alpha$ and $\beta$ are constant, and $\alpha=b, \beta=d$. Also, $a+b x=0$ for all $x$. Taking $x=0$ we have $a=0$. So, $b x=0$ for all possible choices of $x$, which gives $b=0$ by taking successively $x=\left[\begin{array}{lllll}\mathbf{0} & \cdots & \mathbf{e}_{i} & \cdots & \mathbf{0}\end{array}\right]$ for $i=1, \ldots, n-r$. Similarly from $c+d x=0$ we obtain $c=0$ and $d=0$. So, $B Q=0$ and therefore $B=0$.

The converse is clear.

\section{Remarks and questions}

1. The element $a \hat{a} a$ is always regular, with a reflexive inverse of $\hat{a}$, and the set of regular elements is given by $\{b \hat{b} b$; all $b\}$.

2. If $\hat{a}$ is a outer inverse of $a$, then so is $\hat{a}+(1-\hat{a} a)(a-a \hat{a} a)^{\wedge}(1-a \hat{a})$.

3. When $A$ is $m \times n$ over $\mathbb{F}$, we know that $A=R\left[\begin{array}{cc}I_{r} & 0 \\ 0 & 0\end{array}\right] S=R E S$, for some invertible $R$ and $S$. As such $\hat{A}=S^{-1} \hat{E} R^{-1}$ is $n \times m$. The class of all outer inverses of $E$ can be found by direct computation as $\left[\begin{array}{l}I \\ \alpha\end{array}\right] H[I, \beta]$, where $H^{2}=H$ and $\alpha, \beta$ are arbitrary.

4. The expression of $u^{-1} E$ in Corollary 2.2 is very similar to the theory of cosets.

5. Can we select $p$ and $q$ such that it is easier to classify $\widehat{(q a p)}$ ?

6. Can we generalize Theorem 3.1 to $R_{n \times n}$ where $R$ is a unit regular ring?

7. Theorem 3.1 shows that over $\mathbb{F}$ there are basically not enough outer inverses for the triple to be trivially invariant, i.e., $B=0$ or $C=0$.

8. What are some of the other classes of outer inverses that can be characterized?

\section{Acknowledgment}

The authors thank an anonymous referee for his/her valuable corrections. 


\section{References}

[1] F. Graybill; Introduction to Matrices with Applications to Statistics, Wadsworth, Belmont, CA 1969, p.124.

[2] R.E. Hartwig; 1-2 Inverses and the Invariance of $B A^{+} C$, Lin. Algebra App. 11 (1975), $27-275$.

[3] R.E. Hartwig; How to order regular elements, Math. Jap. 25 (1980), 1-13.

[4] R.E. Hartwig, J. Luh; Decomposition of an orthogonally complete atomic unit regular ring, 1979, unpublished.

[5] R.E Hartwig, P. Patrício, R. Puystjens; Diagonalizing triangular matrices via orthogonal Pierce decompositions. Linear Algebra Appl. 401 (2005), 381-391.

[6] R.E. Hartwig, P. Patricio, Two by two units, Electron. J. Linear Algebra 27 (2014), 489-503.

[7] C.R. Rao, S. K. Mitra; Generalized inverse of matrices and its applications. John Wiley \& Sons, Inc., New York-London-Sydney, 1971. 